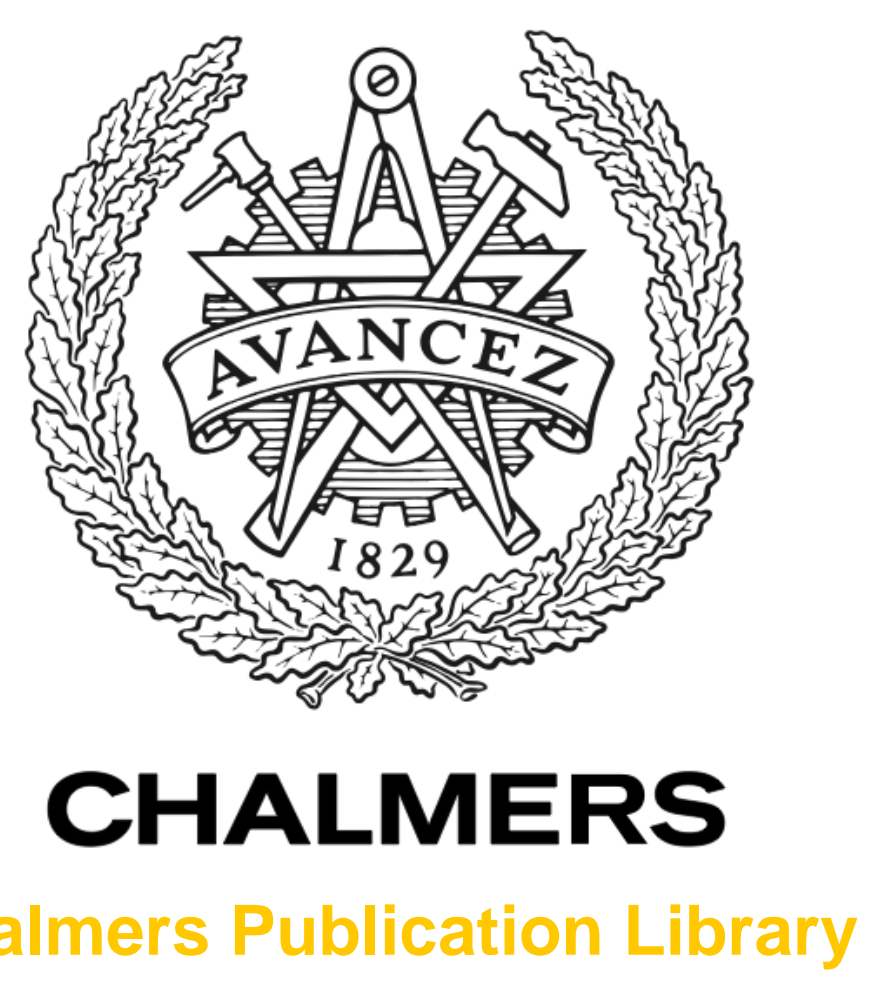

Chalmers Publication Library

\title{
Time-Domain Digital Back Propagation: Algorithm and Finite-Precision Implementation Aspects
}

This document has been downloaded from Chalmers Publication Library (CPL). It is the author's version of a work that was accepted for publication in:

\section{Optical Fiber Communications Conference and Exhibition}

Citation for the published paper:

Fougstedt, C. ; Mazur, M. ; Svensson, L. et al. (2017) "Time-Domain Digital Back Propagation: Algorithm and Finite-Precision Implementation Aspects". Optical Fiber

Communications Conference and Exhibition

Downloaded from: http://publications.lib.chalmers.se/publication/248873

Notice: Changes introduced as a result of publishing processes such as copy-editing and formatting may not be reflected in this document. For a definitive version of this work, please refer to the published source. Please note that access to the published version might require a subscription. 


\title{
Time-Domain Digital Back Propagation: Algorithm and Finite-Precision Implementation Aspects
}

\author{
Christoffer Fougstedt ${ }^{1}$, Mikael Mazur ${ }^{2}$, Lars Svensson $^{1}$, Henrik Eliasson ${ }^{2}$, Magnus Karlsson ${ }^{2}$, \\ and Per Larsson-Edefors ${ }^{1}$ \\ ${ }^{1}$ Dept. of Computer Science and Engineering, ${ }^{2}$ Dept. of Microtechnology and Nanoscience, \\ Chalmers University of Technology, SE-41296 Göteborg, Sweden \\ chrfou@chalmers.se
}

\begin{abstract}
We propose a nonlinear mitigation algorithm designed from an ASIC perspective, and analyze implementation aspects. Given 9 signal and 11 coefficient bits, reach is increased by $105 \%$ compared to linear compensation in single-channel 16-QAM transmission.

OCIS codes: (060.1660) Coherent communications; (060.4510) Optical communications
\end{abstract}

\section{Introduction}

Fiber nonlinearities are a major limiting factor in today's fiber-optic communication links, and effective, implementable mitigation of nonlinear effects is therefore an important research topic. Digital back propagation (DBP) is one of the more popular digital signal processing (DSP) algorithms addressing the issue of fiber nonlinearities [1], and while there are a wealth of papers investigating different variations of DBP algorithms as well as their limitations in WDM systems (see [2] and its references), the only presented ASIC implementation uses high-level synthesis to estimate chip area [3], and does not analyze internal algorithm properties relevant to ASIC implementations.

In this paper, we turn the table; we use a single-channel setup where DBP is known to perform very well, and study the limitations of DBP from an ASIC implementation perspective. We propose a new algorithm, Time-Domain DBP (TD-DBP), and analyze the impact of finite-precision aspects such as quantization and simplified implementation of the nonlinear function.

\section{Time-domain digital back propagation (TD-DBP)}

Light propagation in optical fibers is governed by the nonlinear Schrödinger equation [1],

$$
\frac{\partial A}{\partial z}=(\hat{D}+\hat{N}) A=\left(-\frac{j \beta_{2}}{2} \frac{\partial^{2}}{\partial t^{2}}-\frac{\alpha}{2}\right) A+j \gamma|A|^{2} A,
$$

which can only be solved numerically in the general case. Simulation of fiber-optic communication in the nonlinear regime generally uses the split-step Fourier method (SSFM), where Eq. 1 is solved by assuming that $\hat{D}$ and $\hat{N}$ can be approximated as if they act independently given a very short propagation step; the short steps are then cascaded to solve for the entire link length. In essence, DBP uses the SSFM to calculate the transmitted signal by simulated propagation of the received signal through a fiber with negated parameters with respect to the communication fiber link [1].

For real-time processing, a DBP algorithm needs to be implemented in a streaming block-wise fashion. While frequency-domain methods, such as overlap-save filtering, are effective for long impulse responses, compensating accumulated dispersion corresponding to the steps taken for a span in DBP can be more efficiently implemented in time-domain [4]. Based on this observation, we propose Time-Domain DBP (TD-DBP), in which we implement the dispersive step using FIR filters. TD-DBP has the additional benefit of not requiring FFTs and IFFTs, avoiding internal signal rounding and twiddle-factor rounding in fixed-point FFTs/IFFTs, which would otherwise lead to both quantization noise and spectral leakage between bins.

In discrete time, a single step of TD-DBP can be formulated as

$$
A(z+\Delta z, t)=\left(A(z, t) * h_{C D C}(\Delta z)\right) \cdot \exp ((\alpha \Delta z) / 2) \cdot \exp \left(-j \Delta z \gamma|A|^{2}\right),
$$

where $h_{C D C}(\Delta z)$ is the impulse response of a discrete-time filter capable of compensating for low accumulated chromatic dispersion (CD) corresponding to the in-span step length in DBP. Here, the least-squares constrainedoptimization (LS-CO) filter [5] is used. Using LS-CO, it is possible to optimize the signal in-band response while controlling the out-of-band response, which is very effective when using spectrally limited pulse-shaping such as rootraised cosine (RRC) pulses. In the ensuing evaluation, we focus on a two steps-per-span (StPS) DBP implementation, using modified logarithmic [6] distribution of the steps, with the adjustment factor $k=0.4$ as in [6]. 


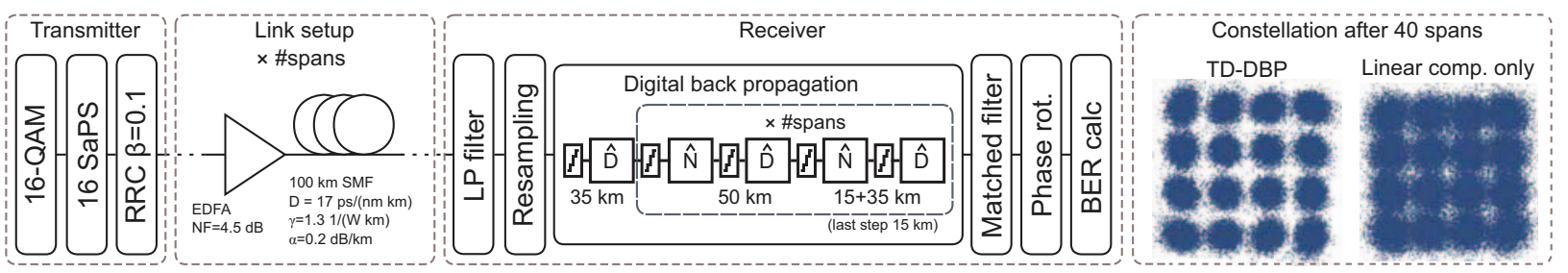

Fig. 1: Block diagram of the simulation setup and constellations after propagation through 40 spans of $100-\mathrm{km}$ SMF at optimal launch power (Sec. 5). (Constellations: TD-DBP uses 9 signal and 11 coefficient bits, but Linear comp. only uses floating-point).

\section{ASIC implementation aspects}

Full floating-point implementation of DSP algorithms for coherent fiber communication systems is not feasible in a real-time ASIC implementation. Additionally, DBP is known to be much more computationally intensive than the real-time algorithms employed now. DBP is fortunately strictly feed-forward and thus, given enough computational resources, it is theoretically possible to reach an arbitrary throughput using parallelization. There are three major ASIC implementation aspects of TD-DBP: signal requantization, coefficient quantization, and implementation of the exponential function. A block diagram of the simulation setup with DBP is shown in Fig. 1, with signal quantization points as shown between the dispersive $(\hat{D})$ and nonlinear $(\hat{N})$ steps. The first and last dispersive step of each cascaded block are combined to reduce the number of filters and quantization errors. The first and last span are thus slightly different; the first uses an additional 35 -km $\hat{D}$-block, and the final $\hat{D}$-block of the last span compensates for $15 \mathrm{~km}$.

Signal requantization: Since DBP requires a large number of cascaded multiplications, the word-length increase for lossless precision is unacceptable. Therefore, internal signal rescaling and rounding is required to counter wordlength growth, which in turn leads to added quantization noise.

Filter coefficient quantization: Unlike signal requantization, coefficient quantization does in itself not result in loss of information, but instead causes a static frequency-response error of the filter, since we are now convolving the signal with a slightly perturbed impulse response. The filter will, thus, have a slight error in phase and amplitude. With the nonlinear term being dependent on the instantaneous value and the requantization being performed after the calculations, the combined effect will be a loss of information.

Exponential function implementation: The standard exponential function does not lend itself to inexpensive hardware implementation. Fortunately, the nonlinear perturbation $\hat{N}$ in Eq. 1 is relatively weak in each step. A few-term Taylor expansion, which is simple to implement in hardware, is therefore likely to be sufficient. In this work, Taylor expansions of orders 1 to 3 are considered for the nonlinear exponential term in Eq. 2.

\section{Simulation setup}

Single-carrier transmission is simulated using the setup shown in Fig. 1. Forward propagation is simulated using SSFM and 16-QAM modulation, $\lambda=1550 \mathrm{~nm}$, RRC pulses at $20 \mathrm{GBd}, 16$ samples-per-symbol (SaPS), and 200 equally distributed StPS. Each span consists of 100-km SMF and an EDFA to compensate for the span loss, with parameters as shown in Fig. 1. The received signal is low-pass filtered, resampled to 2 SaPS, and then back-propagated. Matched filtering is applied, and compensation of static phase rotation is performed before BER calculation. A 2-StPS algorithm is employed as shown in Fig. 1, where each combined filter compensates for $50 \mathrm{~km}$ using 17 taps. The filter is optimized for $60 \%$ of the digital bandwidth, which is chosen with respect to the spectral width of the RRC pulses, with some margin to account for nonlinear spectral broadening. Requantization is performed before each dispersive and nonlinear step, as indicated in Fig. 1.

\section{Results and discussion}

The optimal launch power in the system was found to be approximately $-4 \mathrm{dBm}$ using $\mathrm{CD}$ compensation only, and $1 \mathrm{dBm}$ using 2-StPS DBP. The optimal launch power does not vary significantly with quantization, since the quantization error is independent of launch power and therefore does not change the relation between the error caused by the nonlinearity and amplified spontaneous-emission noise. Taylor expansion of the exponential term was found not to significantly change the optimal launch power, since the nonlinear effects are relatively weak, at each algorithm step, at optimal launch power. As a reference case, we have implemented frequency-domain DBP (FD-DBP) using the same StPS and step distribution as in the TD-DBP case. In the floating-point case, the 17-tap TD-DBP reduces the reach by 3 spans compared to FD-DBP, at a BER of $10^{-3}$ as shown in Fig. 2a. Increasing the compensation bandwidth and the number of taps allows for the same performance as FFT-based DBP, at the cost of increased complexity. 


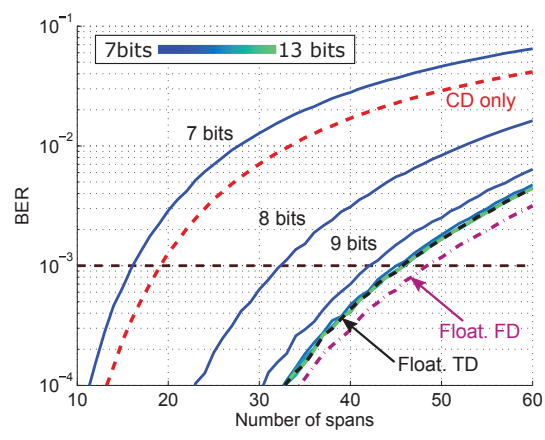

(a) Requantized signal.

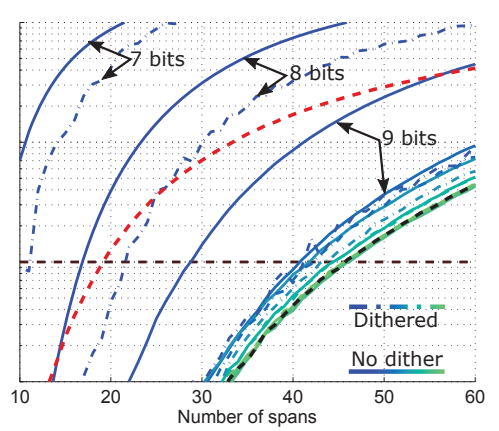

(b) Quantized coefficients.

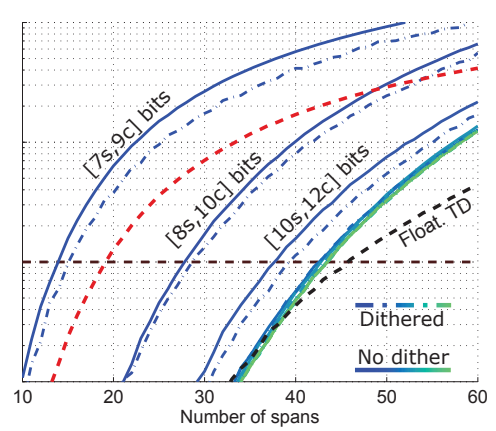

(c) Combined impairments.

Fig. 2: BER as a function of number of $100-\mathrm{km}$ spans for the considered configurations.

Signal requantization: Fig. 2a shows the impact of internal signal rescaling and rounding at word-lengths of 7-13 bits. An internal resolution of at least 8 bits is required in order to outperform floating-point compensation of CD only. Increasing the word-length up to 9 bits gives a significant improvement, while 10 and beyond give diminishing returns.

Filter coefficient quantization: Fig. 2 b shows the impact of coefficient quantization at word-lengths of 7-13 bits. DBP is more sensitive to coefficient quantization than to signal quantization, in contrast to stand-alone CD compensation where the opposite is true [4]. This is because the errors correlate across all spans, giving significant build-up of errors. This was confirmed by dithering the coefficients before quantization with uniform noise having a magnitude of 1 unit of least precision, and thus reducing the correlation between filters. The dot-dashed curves in Fig. $2 \mathrm{~b}$ show the result for the dithered coefficients, where a large improvement is observed for low-resolution coefficients. The jaggedness of the curves is an artifact of the random nature of dithering.

Exponential function implementation: Compared to the ideal exponential, Taylor approximations of orders 2 and 3 give close-to-indistinguishable changes in BER. A first-order expansion $(\exp (z) \approx 1+z)$ was found to improve performance at propagation less than 35 spans and reduce performance above, causing a 3-span reach reduction at a BER of $10^{-3}$. This behavior is caused by the slight signal gain in the first-order approximation, effectively resulting in a per-span slightly increasing $\gamma$. Normalizing the gain of all approximations results in all having negligible performance reduction in terms of BER, compared to the full exponential function.

Combined impairments: Fig. 2c shows the resulting BER as a function of number of spans when both signal and filter coefficients are quantized, with the $\hat{N}$-block implemented using a first-order Taylor approximation. As shown in Fig. 2c, the coefficient word-length (c) is consistently set 2 bits longer than the signal word-length (s). A performance decrease can be observed even at large word-lengths, compared to Fig. 2a and Fig. 2b. This is caused by the slight signal gain in the first-order Taylor approximation.

\section{Conclusion}

We propose a new DBP algorithm, TD-DBP, which is suitable for ASIC implementation. We have analyzed the sensitivity to quantization errors both in signal and filter coefficients, and errors due to approximations, essential for hardware implementation, in the nonlinear step. While the correlated error due to coefficient quantization is an issue, dithering coefficients before quantization was found to give significant improvement. Co-optimizing quantization of the cascaded filters is therefore likely to give further improvement. For a single-channel system with a target BER of $10^{-3}$, TD-DBP using 8 signal and 10 coefficient bits improves the reach by $52 \%$ in comparison to CD compensation only, while using 9 signal and 11 coefficient bits allows us to more than double $(+105 \%)$ the reach.

This work was financially supported by the Knut and Alice Wallenberg Foundation.

\section{References}

[1] E. Ip and J. Kahn, "Compensation of dispersion and nonlinear impairments using digital backpropagation,” IEEE JLT 26, 3416-3425 (2008).

[2] R. Dar and P. Winzer, "On the limits of digital back-propagation in fully loaded WDM systems," IEEE PTL 28, 1253-1256 (2016).

[3] V. Parahyba et al., "Performance against implementation of digital backpropagation for high-speed coherent optical systems," IET EL 51, 1094-1096 (2015).

[4] C. Fougstedt et al., "Power-efficient time-domain dispersion compensation using optimized FIR filter implementation," in "OSA SPPCom," (2015), p. SpT3D.3.

[5] A. Sheikh et al., "Dispersion compensation FIR filter with improved robustness to coefficient quantization errors," IEEE JLT PP (2016).

[6] J. Zhang et al., "Digital nonlinear compensation based on the modified logarithmic step size," IEEE JLT 31, 3546-3555 (2013). 